\title{
Plasma total bilirubin levels predict amputation events in type 2 diabetes mellitus: the Fenofibrate Intervention and Event Lowering in Diabetes (FIELD) study
}

\author{
K. H. Chan • R. L. O'Connell • D. R. Sullivan • \\ L. S. Hoffmann - K. Rajamani • M. Whiting • \\ M. W. Donoghoe • M. Vanhala • A. Hamer • B. Yu • \\ R. Stocker • M. K. C. Ng • A. C. Keech • \\ on behalf of the FIELD study investigators
}

Received: 31 May 2012 / Accepted: 10 December 2012 / Published online: 17 January 2013

(C) Springer-Verlag Berlin Heidelberg 2013

\begin{abstract}
Aims/hypothesis Bilirubin has antioxidant and antiinflammatory activities. Previous studies demonstrated that higher bilirubin levels were associated with reduced prevalence of peripheral arterial disease (PAD). However, the relationship between bilirubin and lower-limb amputation, a consequence of PAD, is currently unknown. We hypothesised that, in patients with type 2 diabetes, bilirubin concentrations may inversely associate with lower-limb amputation.
\end{abstract}

M. K. C. Ng and A. C. Keech are joint senior authors of this study

Electronic supplementary material The online version of this article (doi:10.1007/s00125-012-2818-4) contains peer-reviewed but unedited supplementary material, which is available to authosized users.

K. H. Chan · K. Rajamani • M. K. C. Ng • A. C. Keech Department of Cardiology, Royal Prince Alfred Hospital, Sydney, NSW, Australia

K. H. Chan • B. Yu • M. K. C. Ng • A. C. Keech

Sydney Medical School, Sydney, NSW, Australia

K. H. Chan • M. K. C. Ng

The Heart Research Institute, Sydney, NSW, Australia

K. H. Chan $(\bowtie) \cdot$ R. L. O’Connell • D. R. Sullivan •

K. Rajamani $\cdot$ M. W. Donoghoe • A. C. Keech $(\bowtie)$

National Health and Medical Research Council Clinical Trials

Centre, Level 6, Medical Foundation Building, K25,

University of Sydney, Sydney, NSW 2006, Australia

e-mail: kimhoe_chan@yahoo.com.au

e-mail: tony@ctc.usyd.edu.au

D. R. Sullivan

Department of Biochemistry, Royal Prince Alfred Hospital,

Sydney, NSW, Australia
Methods The relationship between baseline plasma total bilirubin levels and amputation events was analysed in 9,795 type 2 diabetic patients from the Fenofibrate Intervention and Event Lowering in Diabetes (FIELD) study. The analysis plan was pre-specified. Lower-limb amputation was adjudicated blinded to treatment allocation. Relevant clinical and biochemical data were available for analyses. Amputation was a pre-specified tertiary endpoint.

Results Bilirubin concentrations were significantly inversely associated with lower-limb amputation, with a greater than

L. S. Hoffmann · R. Stocker

Center for Vascular Research, School of Medical Sciences

(Pathology) and Bosch Institute, University of Sydney,

Sydney, NSW, Australia

M. Whiting

Department of Medical Biochemistry, Flinders Medical Centre,

Adelaide, SA, Australia

M. Vanhala

Unit of Family Practice, Central Finland Central Hospital,

Jyväskylä, Finland

A. Hamer

Department of Cardiology, Nelson Hospital, Nelson, New Zealand

B. Yu

Department of Molecular and Clinical Genetics,

Royal Prince Alfred Hospital, Sydney, NSW, Australia 
threefold risk gradient across levels. Individuals with lower bilirubin concentrations had a higher risk for first amputation (HR 1.38 per $5 \mu \mathrm{mol} / 1$ decrease in bilirubin concentration, $95 \%$ CI 1.07, 1.79, $p=0.013$ ). The same association persisted after adjustment for baseline variables, including age, height, smoking status, $\gamma$-glutamyltransferase level, $\mathrm{HbA}_{1 \mathrm{c}}$, trial treatment allocation (placebo vs fenofibrate), as well as previous PAD, non-PAD cardiovascular disease, amputation or diabetic skin ulcer, neuropathy, nephropathy and diabetic retinopathy (HR 1.38 per $5 \mu \mathrm{mol} / 1$ decrease in bilirubin concentration, 95\% CI 1.05, 1.81, $p=0.019$ ).

Conclusions/interpretation Our results identify a significant inverse relationship between bilirubin levels and total lowerlimb amputation, driven by major amputation. Our data raise the hypothesis that bilirubin may protect against amputation in type 2 diabetes.

Keywords Amputation $\cdot$ Bilirubin $\cdot$ Fenofibrate Intervention and Event Lowering in Diabetes (FIELD) study . Peripheral arterial disease · Type 2 diabetes mellitus

\begin{tabular}{|c|c|}
\hline \multicolumn{2}{|c|}{ Abbreviations } \\
\hline CVD & Cardiovascular disease \\
\hline FIELD & $\begin{array}{l}\text { Fenofibrate Intervention and Event Lowering } \\
\text { in Diabetes }\end{array}$ \\
\hline GGT & $\gamma$-Glutamyltransferase \\
\hline HMOX-1 & Haem oxygenase-1 \\
\hline IRR & Incidence rate ratio \\
\hline PAD & Peripheral arterial disease \\
\hline UGT1A1 & $\begin{array}{l}\text { Uridine diphosphate glucuronosyltransferase- } 1 \\
\text { family polypeptide A1 }\end{array}$ \\
\hline
\end{tabular}

\section{Introduction}

Non-traumatic lower-limb amputations are often the endstage clinical events in intractable limb ischaemia. Despite modern therapy, peripheral arterial disease (PAD) remains a major burden on the healthcare system, with at least one amputation due to diabetes occurring every $30 \mathrm{~s}$ worldwide, up to eight million patients in the USA being devastated by immobility and significant morbidity, and an annual cost exceeding US $\$ 1.6$ billion in $2001[1,2]$. Furthermore, attention to classic vascular risk factors has failed to substantially reduce the risk of amputation [3], highlighting the need to find novel predictors and biomarkers for PAD and amputation events that may help to identify new therapeutic targets [4-6].

Bilirubin, a product of haem degradation, may confer vascular protective effects [7, 8], and is therefore a possible candidate biomarker for predicting amputation events. Experimental studies have reported that bilirubin possesses potent antioxidant and anti-inflammatory properties in vitro and in vivo [9-14]. Given that atherosclerosis and ischaemia are characterised by a state of heightened inflammation and oxidation $[15,16]$, it is conceivable that bilirubin may confer beneficial effects through these known activities. Consistent with this notion, case-control studies have reported that individuals with elevated bilirubin levels caused by Gilbert's syndrome have a decreased incidence of atherosclerotic disease compared with normal controls $[12,14]$. Also, previous studies reported an inverse relationship between bilirubin levels and PAD prevalence [17-20]. However, the association between bilirubin and the hard clinical endpoint of amputation events has not been reported, to our knowledge. We therefore hypothesised that plasma bilirubin concentrations may inversely associate with lower-limb amputation. We studied this association in a longitudinal cohort of individuals with type 2 diabetes, as these patients have an eightfold higher amputation risk compared with non-diabetic patients [21].

\section{Methods}

Study participants The relationship between baseline plasma total bilirubin levels and non-traumatic amputation was analysed in 9,795 type 2 diabetic patients from the FIELD (Fenofibrate Intervention and Event Lowering in Diabetes) study in a subsidiary analysis. In brief, patients in the FIELD study were randomised to either fenofibrate or placebo treatment between February 1998 and November 2000, and were followed up for a median duration of 5 years [22]. All patients were aged 50-75 years and had a diagnosis of type 2 diabetes according to WHO criteria [23]. Individuals with renal impairment, chronic liver disease, symptomatic gallbladder disease, or those who had experienced a cardiovascular event within the 3 months before recruitment were excluded. Non-traumatic amputation was a pre-specified tertiary endpoint. All amputations that occurred during study follow-up (on-study amputations) were adjudicated blinded to treatment allocation by two clinicians separately, and any discrepancies were resolved by mutual agreement. Pre-study amputations were adjudicated in the same fashion. Major amputations were defined as those above the ankle and minor amputations as those below the ankle [3]. All patients provided written informed consent. This study had ethics committee approval in accordance with the Declaration of Helsinki and Good Clinical Practice Guidelines. The original trial was registered with the International Standard Randomised Controlled Trial Number (ISRCTN) 64783481.

Laboratory measurements Early-morning fasting baseline blood specimens were obtained in all individuals prior to study randomisation. Plasma (EDTA) levels of total 
bilirubin, $\mathrm{HbA}_{1 \mathrm{c}}$ and $\gamma$-glutamyltransferase (GGT) were determined using an automated analyser (Hitachi 917, Roche Diagnostics, Basel, Switzerland). Total bilirubin was measured as the plasma concentration expressed in $\mu \mathrm{mol} / \mathrm{l}$ using the Diazo method [24].

Baseline patient variables A medical history was obtained to determine whether the patient had been diagnosed with or experienced any of: claudication or PAD; prior amputation; diabetic skin ulcer; diabetic retinopathy; neuropathy; or cardiovascular disease (CVD). Age was categorised into $<65$ years or $\geq 65$ years. Previous PAD was present if the patient was diagnosed with or experienced claudication or PAD, and/or previous peripheral revascularisation. Prior non-PAD CVD was defined as any history of CHD or stroke. Neuropathy was present if the patient was diagnosed with or experienced diabetic neuropathy, and/or the patient's foot had absent sensation on monofilament testing. Nephropathy was defined by the presence of albuminuria as previously described [3].

Statistical analyses All analyses were performed on an intention-to-treat basis. Baseline characteristics were analysed with $\chi^{2}$ tests for categorical variables, $t$ tests for continuous variables or, if the distribution of the data was non-normal, the Wilcoxon rank-sum test. Cox proportional hazards regression was used to compute HRs and $95 \%$ CIs to assess the relationship between total bilirubin levels and time to first amputation. As there were 190 amputations occurring in 115 patients, a multiple-event analysis was performed using Poisson regression modelling for all amputation events and adjusting for months of observation and overdispersion, using the Pearson method. The computed HR (or incidence rate ratio [IRR] for the Pearson method) for the bilirubin effect was expressed as the increased risk per $5 \mu \mathrm{mol} / 1$ decrease in bilirubin $(\approx 1 \mathrm{SD}$, a statistically relevant difference in bilirubin concentrations).

A multivariable analysis was also performed adjusting for the following baseline covariates: age; height; smoking status; $\mathrm{HbA}_{1 c}$; history of previous PAD; amputation or diabetic skin ulcer; neuropathy; nephropathy; diabetic retinopathy; and trial treatment allocation (placebo vs fenofibrate). These covariates (which were determined by use of backwards selection and then confirmed by exhaustive search methods) were selected as they have been reported to be significant predictors of first amputation in the FIELD study [3]. Other prior CVD (non-PAD) was also included as a covariate, as this is a well-recognised risk factor for PAD and amputation [25]. Liver function test variables (alanine transaminase and GGT) and alcohol intake were also considered as covariates, but GGT was the only significant predictor of amputation and was therefore retained as a covariate in the multivariable model. The possibility of over-fitting because of the large number of potential predictors assessed and the small number of events was examined by calculating the heuristic shrinkage factor. The shrinkage factor of 0.92 indicates that the degree of over-fitting was negligible. A test of interaction was conducted between bilirubin level and treatment allocation. The cumulative risk curves of time to first amputation across five ordered groups of bilirubin level (categorised according to $5 \mu \mathrm{mol} / 1[\approx 1 \mathrm{SD}]$ increments in bilirubin concentration, i.e. $0-5,6-10,11-15$, $16-20$ and $\geq 21 \mu \mathrm{mol} / \mathrm{l})$ was calculated using the KaplanMeier method, and the $p$ value computed using the logrank test of trend. The cumulative rate of amputation across the five ordered groups of bilirubin levels was analysed using the Cochran-Armitage trend test (after testing for linearity). The test for deviation from linearity was conducted by fitting a model including both a linear and a categorical version of the variable-grouped bilirubin level. For this method, the test of the overall effect of the individual categories assesses the significance of a non-linear component [26]. These tests were performed for both a logistic regression (Cochran-Armitage trend test) and a Cox model (logrank trend test) and indicated no deviation from linearity.

Post hoc analyses were also undertaken according to quintile of bilirubin concentration. A test of trend was performed by fitting a Cox model including a discrete variable derived using the median bilirubin level of each quintile (as the ranges of bilirubin level within each quintile were not equal); the level of significance was based on the score test provided by the model. Deviation from linearity was tested using the method described above. A Cox model was also fitted that included the bilirubin quintile group as a categorical variable, both unadjusted and adjusted for the variables mentioned above. The lowest bilirubin quintile was used as the reference category. Analyses were also performed separately for major and minor amputations, sex and smoking status, but in each case, there were insufficient events in at least one subgroup to perform adjusted analyses.

The stability of bilirubin level over time was assessed by calculation of the reliability ratio in a subset of 923 individuals, with repeat measures at year 1 . The reliability ratio indicates the proportion of overall variation which is true variation as opposed to true plus random variation (i.e. it gives the ratio of the variance of true to observed bilirubin concentration). Hypotheses and the substudy plan were specified prior to data analyses. The substudy analysis plan stipulated the use of the same statistical methods as in the earlier FIELD amputation study [3], and that the five ordered groups of bilirubin were categorised according to $\approx 1$ SD increments in bilirubin concentration. As only five patients, none of whom had undergone amputation, had any missing data, no statistical adjustment was made for 


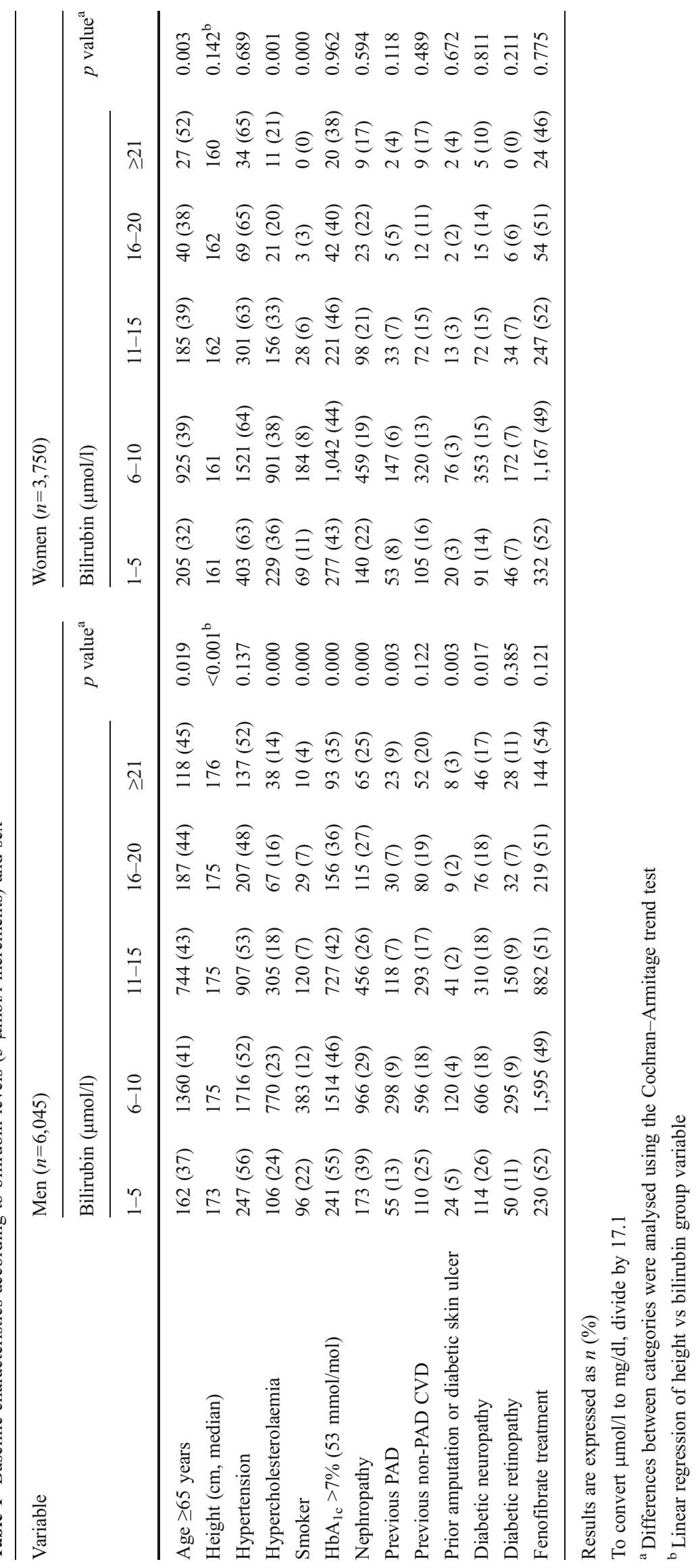




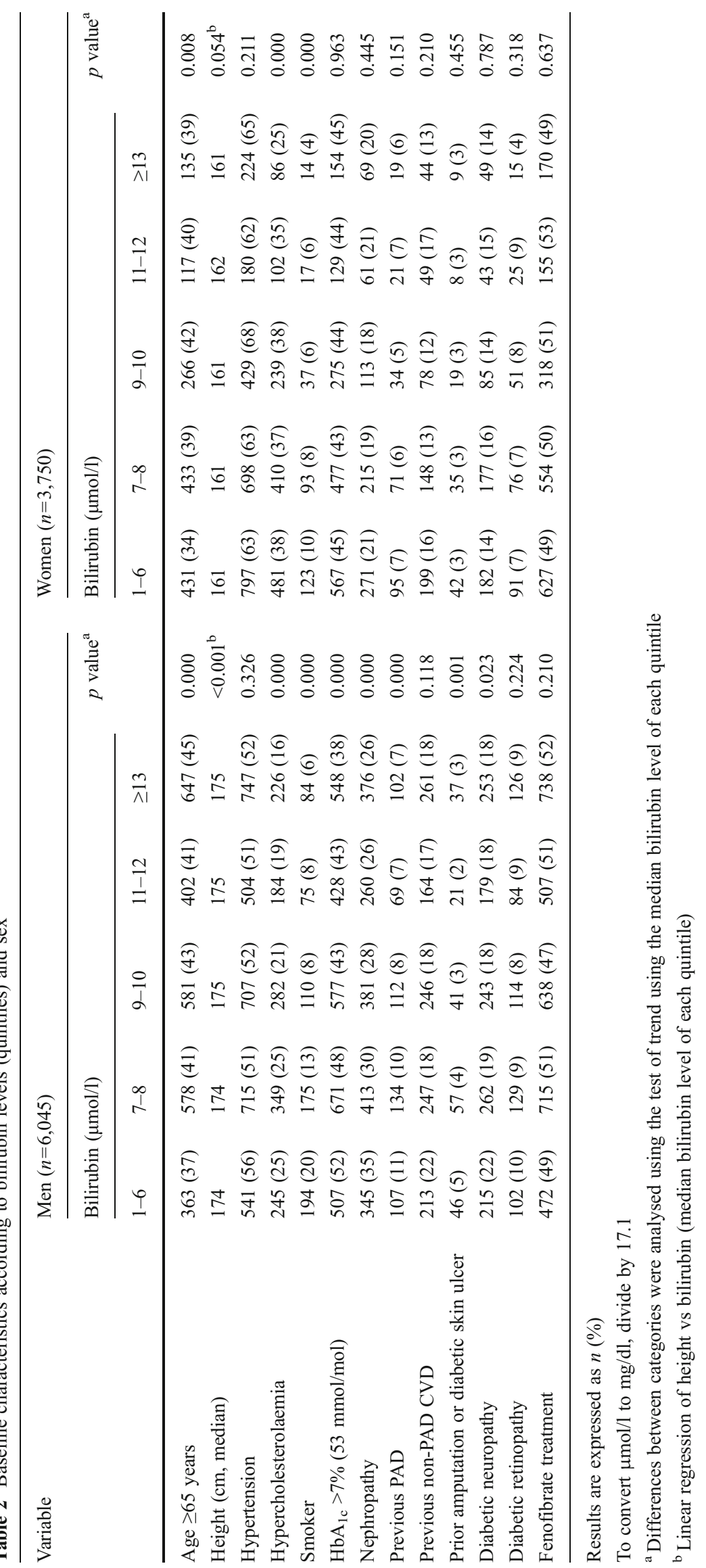


Table 3 Association between baseline plasma total bilirubin concentration and risk of first amputation event, and total amputation events

\begin{tabular}{lll}
\hline Variable & $\begin{array}{l}\text { Risk of amputation (HR per } 5 \mu \mathrm{mol} / 1 \\
\text { decrease in bilirubin, } 95 \% \mathrm{CI})\end{array}$ & $p$ value
\end{tabular}

\begin{tabular}{ccc}
\hline First amputation event & \\
Unadjusted & $1.38(1.07,1.79)$ & 0.013 \\
Adjusted $^{\mathrm{a}}$ & $1.38(1.05,1.81)$ & 0.019 \\
Total amputation & events & \\
Unadjusted & $1.38(1.02,1.86)$ & 0.035 \\
Adjusted $^{\mathrm{b}}$ & $1.37(1.08,1.73)$ & 0.009 \\
\hline
\end{tabular}

To convert $\mu \mathrm{mol} / 1$ to $\mathrm{mg} / \mathrm{dl}$, divide by 17.1

${ }^{a}$ Adjusted for the following baseline patient variables: age, height, smoking status, GGT, $\mathrm{HbA}_{1 \mathrm{c}}$, and history of previous PAD, non-PAD CVD, amputation or diabetic skin ulcer, neuropathy, nephropathy and diabetic retinopathy, as well as trial treatment allocation (placebo vs fenofibrate)

${ }^{\mathrm{b}}$ Poisson method: the IRR, analogous to the HR, is shown

this. A two-sided $p$ value $<0.05$ was considered to indicate statistical significance.

\section{Results}

The FIELD study enrolled 9,795 individuals, with only 22 being lost to follow-up and nine withdrawing from the study. The mean age of the participants was $62 \pm 7$ years, with a median diabetes duration of 5 years (interquartile range 2-10 years). There were 190 amputations occurring in 115 patients, with 93 out of $6,045(1.5 \%)$ men and 22 out of $3,750(0.6 \%)$ women suffering from $\geq 1$ amputation. A total of 35 patients experienced major amputations only, 65 minor amputations only, and 15 one or more of both categories. Baseline patient characteristics according to bilirubin level, sex, and amputation classification are shown in Tables 1 and 2 and electronic supplementary material (ESM) Table 1, respectively. Bilirubin levels were well balanced by study treatment group. The mean baseline plasma total bilirubin concentration was $9.7 \mu \mathrm{mol} / 1$ (median 9.0, SD 4.6, range 1-70 $\mu \mathrm{mol} / 1$ ). In a subset of 923 patients, bilirubin concentration measured 1 year apart did not change significantly, with a mean difference of only $0.3 \mu \mathrm{mol} / 1$ between measurements and a reliability ratio of 0.73 .

Association between baseline plasma total bilirubin concentrations and amputation events Baseline plasma total bilirubin concentrations were significantly inversely associated with lower-limb amputation rate (HR 1.38 per $5 \mu \mathrm{mol} / 1$ decrease in bilirubin concentration, 95\% CI 1.07, $1.79, p=0.013$; Table 3). The same association persisted after adjustment for baseline covariates including age, height, smoking status, GGT, $\mathrm{HbA}_{1 \mathrm{c}}$, trial treatment allocation (placebo vs fenofibrate), and previous PAD, non-PAD CVD, amputation or diabetic skin ulcer, neuropathy, nephropathy and diabetic retinopathy (HR 1.38 per $5 \mu \mathrm{mol} / \mathrm{l}$ decrease in bilirubin concentration, $95 \%$ CI $1.05,1.81, p=0.019)$. There was also no interaction between bilirubin concentration and treatment allocation $(p=0.74)$. Likewise, the multiple-event analysis revealed a similar relationship (IRR 1.38 per $5 \mu \mathrm{mol} / 1$ decrease in bilirubin concentration, $95 \%$ CI 1.02 , $1.86, p=0.035$ ). This association also persisted after adjustment for baseline covariates (IRR 1.37 per $5 \mu \mathrm{mol} / 1$ decrease in bilirubin concentration, 95\% CI 1.08, 1.73, $p=0.009$ ). Patients with only one amputation and multiple $(\geq 2)$ amputation events had similar bilirubin concentrations (median $8 \mu \mathrm{mol} / \mathrm{l}$ for both, $p=0.29$ ).

When the bilirubin levels were categorised into $5 \mu \mathrm{mol} / 1$ $(\approx 1 \mathrm{SD})$ increments in concentration, the cumulative risk of
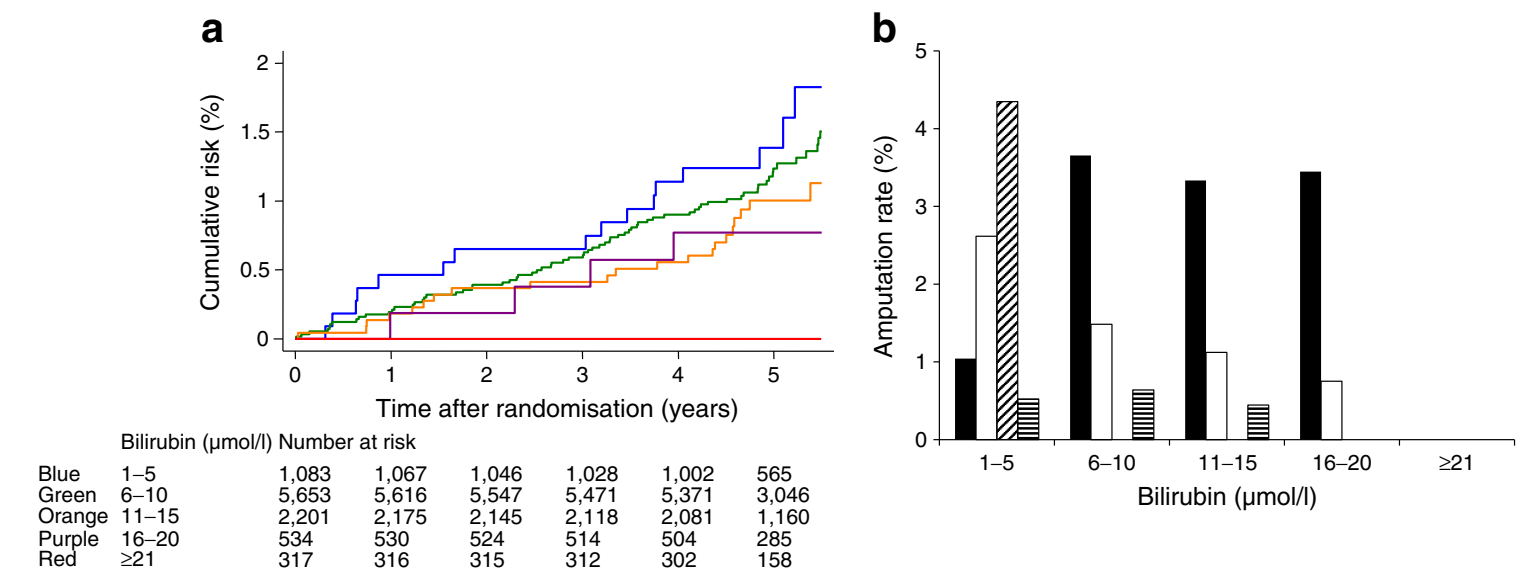

Fig. 1 Amputation risk according to plasma total bilirubin concentration $(5 \mu \mathrm{mol} / 1$ increments). (a) Cumulative risk curves ( $\%$ events over time) to first amputation event. Logrank test of trend, $p=0.025$. (b) Cumulative 5 year amputation rates by sex and smoking status

classification. Black bars, male smokers; white bars, male nonsmokers; diagonally hatched bars, female smokers; horizontally hatched bars, female non-smokers. To convert $\mu \mathrm{mol} / \mathrm{l}$ to $\mathrm{mg} / \mathrm{dl}$, divide by 17.1 
Table 4 Association between baseline plasma total bilirubin concentration (according to quintiles) and risk of first amputation event

\begin{tabular}{lll}
\hline Bilirubin $(\mu \mathrm{mol} / 1)$ & $\begin{array}{l}\text { Risk of first amputation } \\
(\mathrm{HR}, 95 \% \mathrm{CI})\end{array}$ & $p$ value \\
\hline $\begin{array}{l}\text { Unadjusted analysis } \\
\quad \leq 6\end{array}$ & 1 (reference) & - \\
$7-8$ & $0.76(0.47,1.24)$ & 0.269 \\
$9-10$ & $0.71(0.42,1.21)$ & 0.206 \\
$11-12$ & $0.71(0.38,1.32)$ & 0.284 \\
$\geq 13$ & $0.51(0.27,0.94)$ & 0.031 \\
Adjusted analysis ${ }^{\mathrm{a}}$ & & \\
$\leq 6$ & $1($ reference $)$ & 0.244 \\
$7-8$ & $0.74(0.45,1.22)$ & 0.158 \\
$9-10$ & $0.67(0.39,1.16)$ & 0.400 \\
$11-12$ & $0.76(0.39,1.45)$ & 0.035 \\
$\geq 13$ & $0.50(0.27,0.95)$ &
\end{tabular}

To convert $\mu \mathrm{mol} / \mathrm{l}$ to $\mathrm{mg} / \mathrm{dl}$, divide by 17.1

${ }^{a}$ Adjusted for the following baseline patient variables: age, height, smoking status, GGT, $\mathrm{HbA}_{1 \mathrm{c}}$, and history of previous PAD, non-PAD CVD, amputation or diabetic skin ulcer, neuropathy, nephropathy and diabetic retinopathy, as well as trial treatment allocation (placebo vs fenofibrate)

amputation over the study duration was highest in patients with the lowest $(0-5 \mu \mathrm{mol} / \mathrm{l})$ bilirubin concentrations, and lowest (with no amputation events) in patients with the highest bilirubin $(\geq 21 \mu \mathrm{mol} / \mathrm{l})$ concentrations $(p=0.025$; Fig. 1a). Figure 1b shows the cumulative rate of amputation by sex and smoking status. If the patients with bilirubin $\geq 21 \mu \mathrm{mol} / 1$ were excluded from the analysis, the relationship was no longer significant $(p=0.11)$.

Analyses according to bilirubin quintiles also demonstrated a similar inverse association between bilirubin and amputation. A bilirubin level $<6 \mu \mathrm{mol} / 1$ carries a significantly greater (twofold higher) risk than that for individuals with bilirubin $>12 \mu \mathrm{mol} / 1$ (HR 0.51 for highest quintile, 95\% CI 0.27, 0.94, $p=0.031$; Table 4 and Fig. 2a). This association also persisted after adjusting for baseline covariates (HR 0.50, 95\% CI 0.27 , $0.95, p=0.035$ ). Figure $2 b$ shows the cumulative rate of amputation by sex, smoking status and bilirubin quintile.

Interestingly, the inverse association between bilirubin concentration and amputation event was separately significant for major (HR 1.83 per $5 \mu \mathrm{mol} / 1$ decrease in bilirubin concentration, 95\% CI 1.17, 2.87, $p=0.009)$, but not minor (HR 1.22 per $5 \mu \mathrm{mol} / 1$ decrease in bilirubin concentration, 95\% CI 0.92, 1.61, $p=0.18$ ) amputation (ESM Fig. 1). Analyses according to bilirubin quintile also revealed a similar inverse association (ESM Table 2 and ESM Fig. 1).

Analysis according to sex Consistent with previous studies $[4,5,19,27,28]$, our study showed that women had lower median bilirubin concentrations than men ( 8 vs $10 \mu \mathrm{mol} / \mathrm{l})$. We found that the inverse relationship between bilirubin concentrations and risk of first amputation was highly significant for men (HR 1.60 per $5 \mu \mathrm{mol} / 1$ decrease in bilirubin concentration, 95\% CI 1.19, 2.14, $p=0.0017$ ), but not women (HR 1.90 per $5 \mu \mathrm{mol} / 1$ decrease in bilirubin concentration, $95 \%$ CI $0.84,4.33, p=0.126$; $p$ for interaction by sex= 0.71; Fig. 3a-c). Analyses according to bilirubin quintile also demonstrated a similar inverse association for men (HR 0.38 for highest quintile, 95\% CI $0.19,0.75, p=0.005$; Table 5 and Fig. $3 \mathrm{~d}-\mathrm{f}$ ), but not women. The amputation rate among individuals $\geq 65$ years old was higher than in younger individuals of both sexes, with cumulative first amputation rates of $2.1 \%$ vs $1.1 \%$ for men and $1.1 \%$ vs $0.3 \%$ for women, respectively.

Analysis according to smoking status Consistent with previous studies [19, 29], our study showed that current

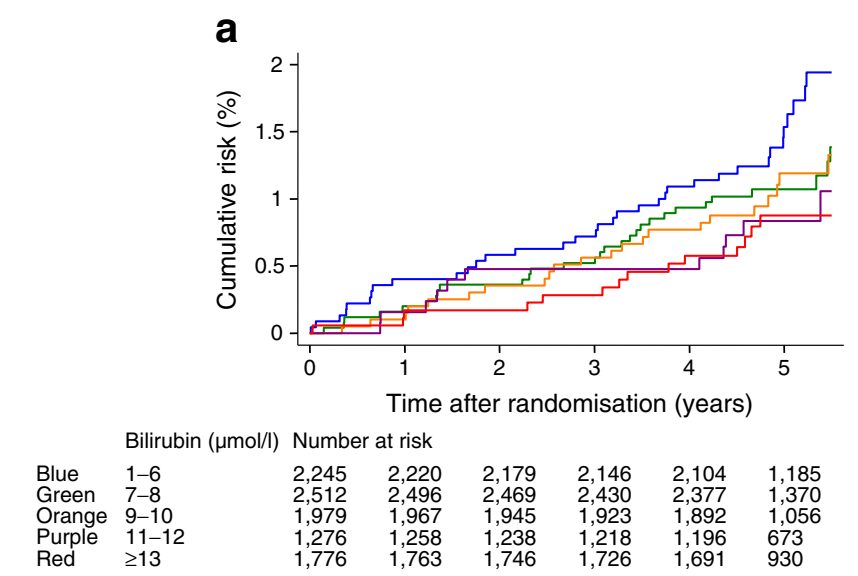

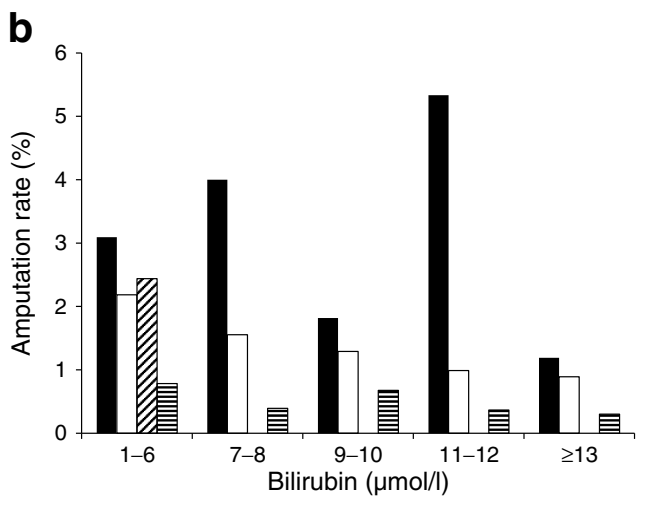

classification. Black bars, male smokers; white bars, male nonsmokers; diagonally hatched bars, female smokers; horizontally hatched bars, female non-smokers. To convert $\mu \mathrm{mol} / \mathrm{l}$ to $\mathrm{mg} / \mathrm{dl}$, divide by 17.1 
a

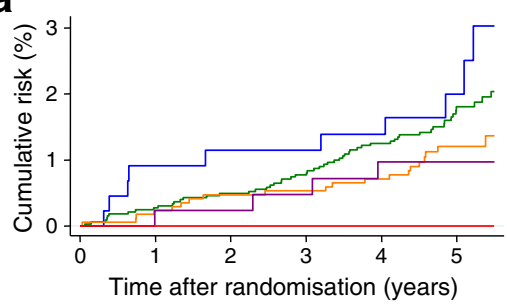

Bilirubin $(\mu \mathrm{mol} / \mathrm{l})$ Number at risk

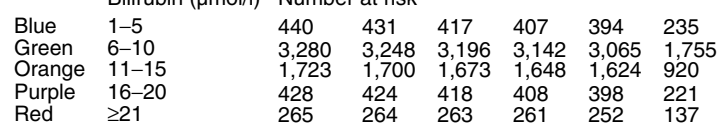

b

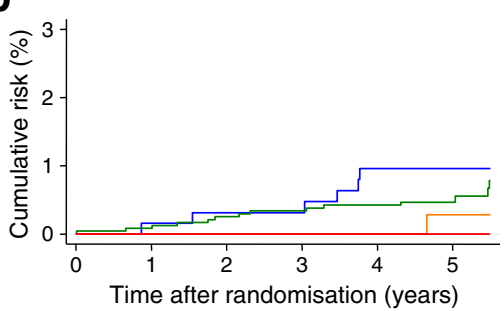

Bilirubin $(\mu \mathrm{mol} / \mathrm{l}) \quad$ Number at risk

$\begin{array}{llllllll}\text { Blue } & 1-5 & 643 & 636 & 629 & 621 & 608 & 330 \\ \text { Green } & 6-10 & 2,373 & 2,368 & 2,351 & 2,329 & 2,306 & 1,291 \\ \text { Orange } & 11-15 & 478 & 475 & 472 & 470 & 457 & 240 \\ \text { Purple } & 16-20 & 106 & 106 & 106 & 106 & 106 & 64 \\ \text { Red } & \geq 21 & 52 & 52 & 52 & 51 & 50 & 21\end{array}$

\section{C}

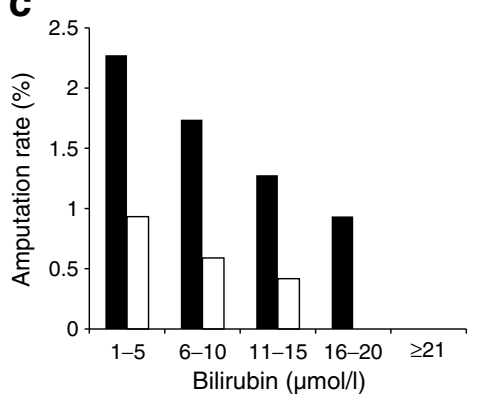

No. of amputations

$\begin{array}{lccccc}\text { Men } & 10 & 57 & 22 & 4 & 0 \\ \text { Women } & 6 & 14 & 2 & 0 & 0\end{array}$

Fig. 3 Association by sex classification. (a, b) Cumulative risk curves (\% events over time) to first amputation event according to baseline plasma total bilirubin concentration $(5 \mu \mathrm{mol} / 1$ increments) in (a) men, and (b) women. Logrank test of trend, $p=0.0045$ for men and $p=0.125$ for women. (c) Cumulative 5 year amputation rates according to baseline plasma total bilirubin concentration $(5 \mu \mathrm{mol} / 1$ increments $)$ in men and women. Cochran-Armitage trend test, $p=0.0041$ for men and $p=0.131$ for women. Black bars, men; white bars, women. (d, e)

smokers had lower median bilirubin levels compared with non-smokers ( 8 vs $9 \mu \mathrm{mol} / \mathrm{l})$. We found that the inverse relationship between bilirubin concentration and risk of first amputation was significant for non-smokers (HR 1.34 per $5 \mu \mathrm{mol} / 1$ decrease in bilirubin concentration, $95 \%$ CI 1.01 , 1.77, $p=0.04$ ), but not for smokers (HR 1.24 per $5 \mu \mathrm{mol} / 1$ decrease in bilirubin concentration, 95\% CI $0.66,2.33, p=$ $0.513 ; p$ for interaction by smoking status $=0.81$; Fig. $4 \mathrm{a}-\mathrm{c}$ ). The same analyses according to bilirubin quintile were not d
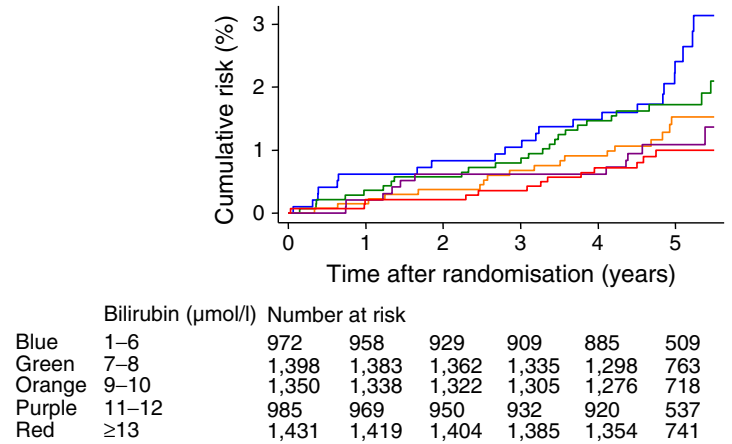

e

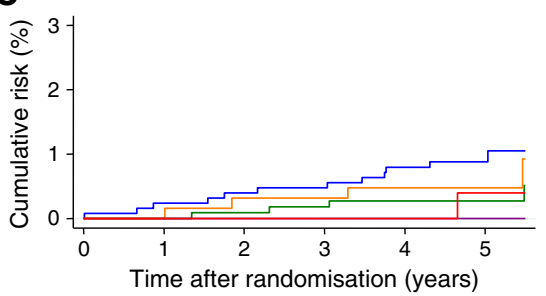

Bilirubin $(\mu \mathrm{mol} / \mathrm{l})$ Number at risk
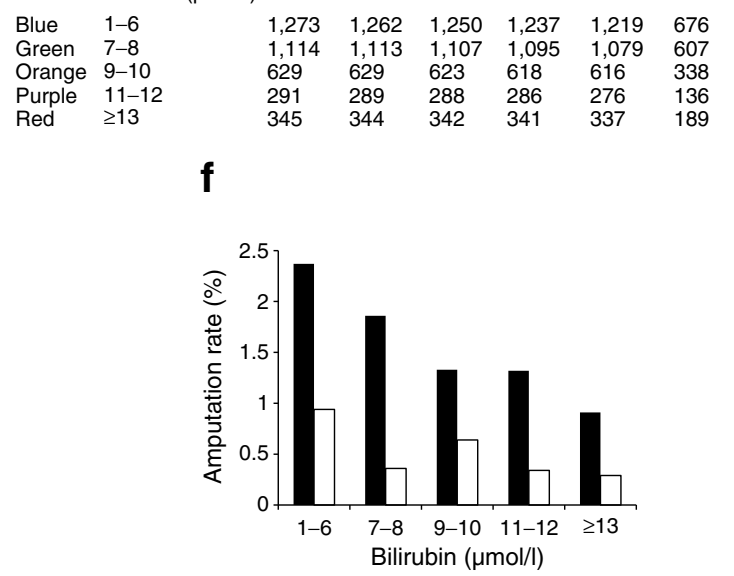

No. of amputations

$\begin{array}{lccccc}\text { Men } & 23 & 26 & 18 & 13 & 13 \\ \text { Women } & 12 & 4 & 4 & 1 & 1\end{array}$

Cumulative risk curves ( $\%$ events over time) to first amputation event according to baseline plasma total bilirubin concentration (quintiles) in (d) men, and (e) women. Logrank test of trend (median method), $p=$ 0.0024 for men and $p=0.136$ for women. (f) Cumulative 5 year amputation rates according to baseline plasma total bilirubin concentration (quintiles) in men and women. Cochran-Armitage trend test (median method), $p=0.0023$ for men and $p=0.136$ for women. Black bars, men; white bars, women. To convert $\mu \mathrm{mol} / \mathrm{l}$ to $\mathrm{mg} / \mathrm{dl}$, divide by 17.1

statistically significant for either non-smokers or smokers, although there was a trend towards an inverse association in non-smokers (Table 6 and Fig. 4d-f).

\section{Discussion}

Our results identify a significant inverse relationship between baseline plasma total bilirubin levels and lower-limb 
Table 5 Association between baseline plasma total bilirubin concentration (quintiles) and risk of first amputation event, by sex

\begin{tabular}{lll}
\hline Bilirubin $(\mu \mathrm{mol} / \mathrm{l})$ & Risk of first amputation $(\mathrm{HR}, 95 \% \mathrm{CI})$ & $p$ value \\
\hline $\begin{array}{ll}\text { Men } \\
\leq 6\end{array}$ & 1 (reference) & - \\
$7-8$ & $0.78(0.44,1.36)$ & 0.377 \\
$9-10$ & $0.56(0.30,1.03)$ & 0.062 \\
$11-12$ & $0.56(0.28,1.10)$ & 0.092 \\
$\geq 13$ & $0.38(0.19,0.75)$ & 0.005 \\
Women & & \\
$\leq 6$ & $1($ reference $)$ & - \\
$7-8$ & $0.37(0.12,1.16)$ & 0.089 \\
$9-10$ & $0.67(0.22,2.07)$ & 0.483 \\
$11-12$ & $0.37(0.05,2.83)$ & 0.337 \\
$\geq 13$ & $0.30(0.04,2.34)$ & 0.253 \\
\hline
\end{tabular}

To convert $\mu \mathrm{mol} / \mathrm{l}$ to $\mathrm{mg} / \mathrm{dl}$, divide by 17.1

amputation in patients with type 2 diabetes. This association was observed between bilirubin and total amputations, as well as separately for major but not minor amputations. The relationship with total (major plus minor) amputation remains robust even after adjusting for numerous other classic vascular and known amputation risk factors, liver function tests (GGT) and study treatment allocation, suggesting that it is likely to be independent of these other factors. Our data are therefore consistent with the hypothesis that bilirubin may be a useful biomarker for amputation in patients with type 2 diabetes. Potentially, this association is of major clinical importance, as the natural range of bilirubin levels seen here covers a striking, greater than threefold, risk gradient in amputation rates over 5 years.

Previous studies focusing on the relationship between bilirubin and PAD also reported a similar inverse association. The largest of these studies analysed 7,075 individuals from the National Health and Nutrition Examination Survey, and reported that increased bilirubin levels were associated with a reduced prevalence of PAD [19]. However, hard clinical endpoints were not explored in that and other similar smaller case-control studies [17-20]. Our study therefore for the first time extends these findings to amputation events. This inverse association is further supported by studies showing a similar relationship between bilirubin levels and CHD, ischaemic stroke, and surrogate markers of atherosclerosis including endothelial dysfunction and carotid intima-media thickness $[12,14,29-42]$. A number of prospective studies found a Uor reversed J-shaped relationship rather than an inverse relationship between bilirubin levels and CHD [4, 5, 27, 28, 30, $33,34,37]$. The differences between our study and these earlier studies are that they concentrated on CHD and mainly enrolled men, although whether these differences could plausibly explain the differing relationships reported is unclear.
Nonetheless, our results, together with the previously published studies, support the possibility that bilirubin may be protective against atherosclerotic disease.

It is well established that atherosclerosis and its consequence, tissue ischaemia, are characterised by a state of heightened inflammation and oxidative stress $[15,16]$. Studies have shown that bilirubin possesses antioxidant and anti-inflammatory activities in vitro $[9,11]$. These experimental studies are supported by human studies reporting that individuals with elevated bilirubin levels due to Gilbert's syndrome have higher antioxidant capacity and lower proinflammatory markers compared with control individuals [13, 14, 42]. Moreover, in a rat model of vascular injury, hyperbilirubinaemic Gunn rats had reduced neointima formation after balloon injury compared with control wild-type animals, suggesting that bilirubin itself may prevent the development of intimal hyperplasia [43]. How relevant these findings are, with much higher bilirubin levels in Gunn rats compared with humans, is still debated. The potential anti-atherogenic properties of bilirubin could also be consistent with our findings that the inverse association with amputation events was significant for major (which may more accurately reflect large-vessel atherosclerotic disease) but not minor amputation.

Our results are consistent with the previous study by Perlstein et al [19], showing a significant inverse relationship between bilirubin levels and amputation in men but not women. The lack of significant association in women may simply reflect the lower amputation rates in women compared with men, and it should be noted that there was no significant interaction by sex, so we were unable to conclude that the relationship between bilirubin and amputation risk truly differs by sex. Similarly (in contrast to the study by Perlstein et al), although we found a significant inverse relationship in non-smokers (when analysed according to $5 \mu \mathrm{mol} / 1$ bilirubin increments), but not smokers, again there was no significant interaction by smoking status, and only $9 \%$ of the cohort were current smokers. An alternative explanation for this disparate result may be that our study cohort exclusively comprised patients with type 2 diabetes, whereby the adverse combination of hyperglycaemia and smoking might negate any antioxidative benefits that bilirubin might otherwise confer in amputation prevention.

Plasma bilirubin concentrations are determined by the relative activities of the enzymes that form and remove bilirubin. Therefore, a better understanding of the pathways that regulate bilirubin concentrations may lead to new therapeutic targets for the treatment of atherosclerotic disease [7]. This is of particular importance to PAD, in which progressive disease leads to amputation at rates that have not changed significantly over the last three decades despite advances in vascular-targeted therapies [2]. The enzymes 
a

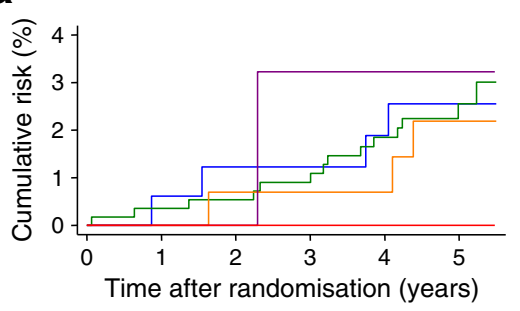

Bilirubin $(\mu \mathrm{mol} / \mathrm{l})$ Number at risk

$\begin{array}{llllllll}\text { Blue } & 1-5 & 165 & 161 & 158 & 153 & 148 & 92 \\ \text { Green } & 6-10 & 567 & 556 & 544 & 530 & 509 & 289 \\ \text { Orange } & 11-15 & 148 & 145 & 141 & 139 & 133 & 75 \\ \text { Purple } & 16-20 & 32 & 32 & 32 & 30 & 30 & 14 \\ \text { Red } & \geq 21 & 10 & 10 & 10 & 10 & 9 & 4\end{array}$

b

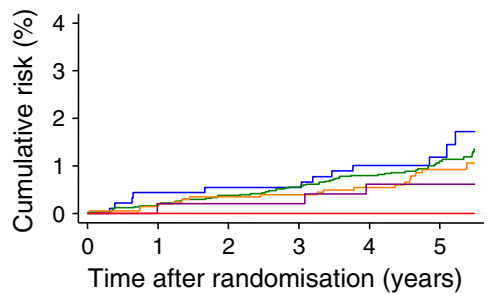

Bilirubin $(\mu \mathrm{mol} / \mathrm{l})$ Number at risk
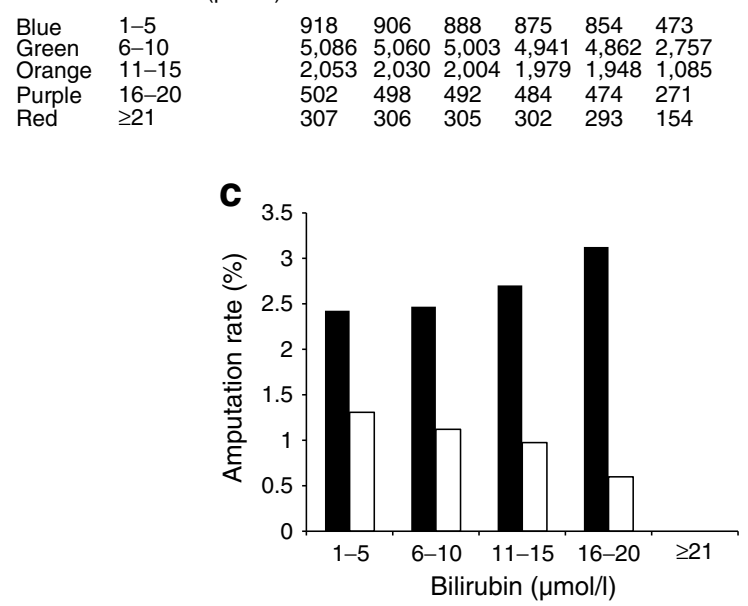

Fig. 4 Association according to smoking status. (a, b) Cumulative risk curves ( $\%$ events over time) to first amputation event according to baseline plasma total bilirubin concentration ( $5 \mu \mathrm{mol} / 1$ increments) in (a) smokers, and (b) non-smokers. Logrank test of trend, $p=0.96$ for smokers and $p=0.034$ for non-smokers. (c) Cumulative 5 year amputation rates according to baseline plasma total bilirubin concentration trend test, $p=0.98$ for smokers and $p=0.032$ for non-smokers. Black bars, smokers; white bars, non-smokers. (d, e) Cumulative risk curves (\% events over time) to first amputation event according to baseline

most widely studied to date include haem oxygenase-1 (HMOX-1) and uridine diphosphate glucuronosyltransferase1 family polypeptide A1 (UGT1A1) [6]. HMOX-1 catalyses the rate-limiting step of haem degradation to carbon monoxide, ferrous iron and biliverdin [44]. Biliverdin is then rapidly reduced to bilirubin via biliverdin reductase. Although initially known for its metabolic role in haem catabolism, it is now well recognised that HMOX-1 may confer vascular protective effects, and this may be mediated, in part, via its haem d

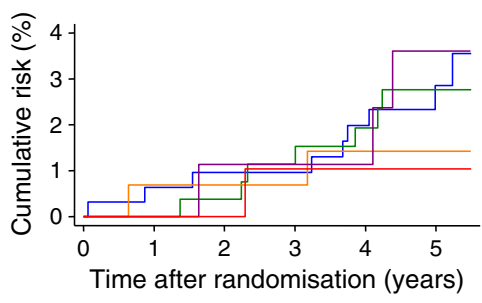

Bilirubin $(\mu \mathrm{mol} / \mathrm{l})$ Number at risk

$\begin{array}{llllllll}\text { Blue } & 1-6 & 317 & 308 & 302 & 295 & 284 & 172 \\ \text { Green } & 7-8 & 268 & 266 & 262 & 253 & 243 & 140 \\ \text { Orange } & 9-10 & 147 & 143 & 138 & 135 & 130 & 69 \\ \text { Purple } & 11-12 & 92 & 89 & 86 & 84 & 80 & 45 \\ \text { Red } & \geq 13 & 98 & 98 & 97 & 95 & 92 & 48\end{array}$

e

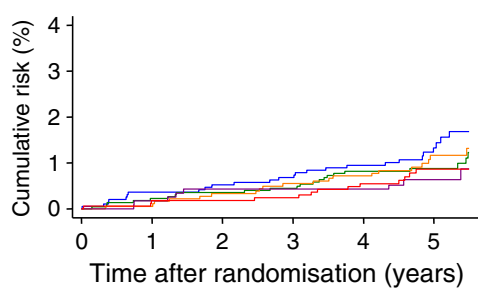

Bilirubin $(\mu \mathrm{mol} / \mathrm{l})$ Number at risk

$\begin{array}{llllllll}\text { Blue } & 1-6 & 1,928 & 1,912 & 1,877 & 1,851 & 1,820 & 1,013 \\ \text { Green } & 7-8 & 2,244 & 2,230 & 2,207 & 2,177 & 2,134 & 1,230 \\ \text { Orange } & 9-10 & 1,832 & 1,824 & 1,807 & 1,788 & 1,762 & 987 \\ \text { Purple } & 11-12 & 1,184 & 1,169 & 1,152 & 1,134 & 1,116 & 628 \\ \text { Red } & \geq 13 & 1,678 & 1,665 & 1,649 & 1,631 & 1,599 & 882\end{array}$

f

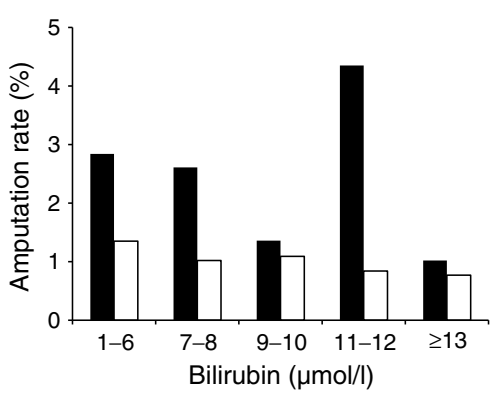

plasma total bilirubin concentration (quintiles) in (d) smokers, and (e) non-smokers. Logrank test of trend (median method), $p=0.58$ for smokers and $p=0.095$ for non-smokers. (f) Cumulative 5 year amputation rates according to baseline plasma total bilirubin concentration (quintiles) in smokers and non-smokers. Cochran-Armitage trend test, $p=0.56$ for smokers and $p=0.09$ for non-smokers. Black bars, smokers; white bars, non-smokers. To convert $\mu \mathrm{mol} / \mathrm{l}$ to $\mathrm{mg} / \mathrm{dl}$, divide by 17.1

catabolism by-products, carbon monoxide and bilirubin [7, 8]. Supporting this, Kawamura et al reported that both HMOX-1 induction and the addition of bilirubin (but not carbon monoxide) conferred anti-inflammatory effects on human endothelial cells in vitro, suggesting that the antiinflammatory properties of HMOX-1 may be mediated through the action of bilirubin [9].

In humans, it is thought that the length of the $(\mathrm{GT})_{n}$ repeat is associated with variation in the HMOXI gene 
Table 6 Association between baseline plasma total bilirubin concentration (quintiles) and risk of first amputation event, by smoking status

\begin{tabular}{lll}
\hline Bilirubin $(\mu \mathrm{mol} / \mathrm{l})$ & Risk of first amputation $(\mathrm{HR}, 95 \% \mathrm{CI})$ & $p$ value \\
\hline $\begin{array}{l}\text { Smoker } \\
\leq 6\end{array}$ & 1 (reference) & - \\
$7-8$ & $0.92(0.34,2.48)$ & 0.875 \\
$9-10$ & $0.50(0.11,2.31)$ & 0.375 \\
$11-12$ & $1.58(0.49,5.14)$ & 0.445 \\
$\geq 13$ & $0.36(0.05,2.82)$ & 0.328 \\
Non-smoker & & \\
$\leq 6$ & $1($ reference $)$ & - \\
$7-8$ & $0.75(0.43,1.32)$ & 0.319 \\
$9-10$ & $0.80(0.45,1.43)$ & 0.455 \\
$11-12$ & $0.63(0.30,1.31)$ & 0.217 \\
$\geq 13$ & $0.57(0.30,1.12)$ & 0.103 \\
\hline
\end{tabular}

To convert $\mu \mathrm{mol} / \mathrm{l}$ to $\mathrm{mg} / \mathrm{dl}$, divide by 17.1

activity, with shorter $(\mathrm{GT})_{n}$ repeats having higher $H M O X 1$ transcriptional activity and expression compared with longer $(\mathrm{GT})_{n}$ repeats [8]. Studies exploring the association between $H M O X 1$ polymorphism, bilirubin levels and vascular events have yielded conflicting results $[6,38,45,46]$. One such study reported that longer $(\mathrm{GT})_{n}$ repeats (and hence lower $H M O X 1$ activity) were associated with lower bilirubin levels and higher risk of CHD [38]. After adjusting for bilirubin, the effect of $H M O X 1$ polymorphism on risk of CHD was no longer present, suggesting that the effect of $H M O X 1$ polymorphism might be mediated through its influence on bilirubin levels [38]. However, other studies have not found such an association between $H M O X 1$ polymorphism and bilirubin $[45,46]$. Likewise, when comparing FIELD participants who developed on-study amputation with those who did not, there was no significant difference in the presence of the L allele (i.e. longer $[\mathrm{GT}]_{n}$ repeats) between these two groups of patients (see the ESM Methods, ESM Figs 2 and 3 and ESM Tables 3-6). These results suggest that carbon monoxide, (i.e. an HMOX1-derived product other than biliverdin/bilirubin), may not be responsible for the decrease in amputation rate associated with high bilirubin levels.

It is increasingly thought that hepatic UGT1A1, rather than HMOX-1, plays a greater role in regulating bilirubin levels [6]. The link between UGT1A1 polymorphism, bilirubin and vascular disease was first noted with the low CHD prevalence in patients with Gilbert's syndrome, a hereditary unconjugated hyperbilirubinaemia secondary to UGT1A1 deficiency [14]. Subsequently, a prospective study involving 1,780 individuals from the Framingham Heart Study Offspring cohort found that UGT1A1 polymorphism resulting in higher bilirubin levels was associated with lower risk of cardiovascular events [36]. Again, however, some other smaller studies have failed to show the same association between UGT1A1 polymorphism and CVD [18, 34, 47]. It is possible that these smaller studies lack the statistical power to detect this association. Nevertheless, clear associations between $U G T 1 A 1$ and bilirubin have been demonstrated in most studies, suggesting that UGT1Al may be a more important factor than $H M O X 1$ in regulation of bilirubin levels [6]. In our patient cohort, it is likely that the 317 (out of 9,795) patients with bilirubin concentration $>20 \mu \mathrm{mol} / 1$ have Gilbert's syndrome, which is consistent with the estimated prevalence of $3-5 \%$ in the general population, although we do not have the results of UGT1A1 polymorphism for confirmation. Therefore, future studies exploring the link between UGT1A1 polymorphism and amputation in the FIELD study cohort will also be important to confirm the results from these previous studies.

There are several limitations of this study. First, bilirubin was, in most cases, only measured once at baseline. It is possible that bilirubin measurements vary significantly for an individual over time. However, random measurement error may result in an underestimation of a true association. Furthermore, in a subset of patients who had their bilirubin remeasured after 1 year, mean bilirubin concentrations had not changed significantly and the individual's levels over 1 year correlated strongly. Second, our study cohort included only patients with type 2 diabetes, and our findings cannot necessarily be generalised to the non-diabetic population. Third, bilirubin levels are inversely associated with glucose levels [48, 49], thereby potentially confounding the results from our study exclusively comprising patients with type 2 diabetes. However, reassuringly, the inverse relationship between bilirubin level and amputation survives adjustment for glycaemic control, and fasting glucose levels were measured at all time points. Fourth, our study does not provide a proven mechanism for the observed association between bilirubin and amputation. Last, the association reported here is not necessarily causal, and could be a false-positive result arising from multiple comparisons, as the FIELD study is exploring risk factors for CHD in diabetes as well as for each of the microvascular complications of diabetes. Therefore, it is possible that the association of bilirubin with amputations is overestimated, and that bilirubin may be a marker rather than an agent directly protective against amputation. Randomised studies raising bilirubin would be better suited to establishing the causal link. This last point is relevant as uric acid, although originally proposed as an antioxidant, is now generally accepted as a biomarker for lifestyle factors rather than a directly protective antioxidant $[5,50]$.

Despite these limitations, the strengths of the current study include its large sample size, longitudinal nature and the very well-characterised nature of the patient cohort. In 
fact, the inverse association between bilirubin level and amputation persisted despite adjusting for a wide range of known risk factors for diabetic complications and amputation (though this did not negate the striking effects of fenofibrate treatment itself to reduce amputation events even after bilirubin adjustment [HR $0.64,95 \%$ CI 0.44 , 0.94, $p=0.02]$ ).

In summary, our study reports for the first time a significant association between higher bilirubin levels and reduced risk of non-traumatic lower-limb amputation in individuals with type 2 diabetes. Furthermore, this association is not explained by other risk factors for amputation in the FIELD study. Our data raise the hypothesis that bilirubin may be a useful biomarker for, and may protect against, amputation in type 2 diabetes. Ultimately, a better understanding of the genes regulating bilirubin level and the potentially protective mechanisms may result in new therapeutic targets for the treatment of PAD and the prevention of amputation in the future.

Acknowledgements The authors acknowledge the advice of S. Curtis (School of Veterinary Sciences, University of Bristol, Bristol, UK) on genotyping techniques. We also thank R. Pike (National Health and Medical Research Council Clinical Trials Centre, University of Sydney, Sydney, NSW, Australia) for her assistance with preparation of the figures and tables.

Funding K. H. Chan is supported by the National Heart Foundation postgraduate scholarship (PC 08S 4127). L. S. Hoffmann was awarded the Feodor-Lynen Fellowship from the Alexander von HumboldtFoundation. Her work was funded in part by a Program Grant from the National Health and Medical Research Council of Australia to R. Stocker. R. Stocker is supported by a National Health and Medical Research Council Senior Principal Research Fellowship. Laboratoires Fournier (now part of Abbott Pharmaceuticals) and the National Health and Medical Research Council funded the FIELD study.

Duality of interest Some authors have been reimbursed by the pharmaceutical industry for the costs of participating in scientific meetings, contributing to advisory boards, or doing other research: D. R. Sullivan (Abbott Pharmaceuticals, AstraZeneca, Merck Sharp and Dohme, Pfizer, sanofi-aventis, Roche and Amgen) and K. Rajamani (Abbott Pharmaceuticals). All other authors declare that there is no duality of interest associated with this manuscript.

Contribution statement $\mathrm{KHC}, \mathrm{LSH}, \mathrm{MV}, \mathrm{AH}, \mathrm{RS}, \mathrm{MKCN}$ and ACK were responsible for the conception and design of the study. KHC, RO'C, DRS, LSH, KR, MW, MKCN and ACK acquired the data; KHC, RO'C, DRS, KR, MWD, BY, MKCN and ACK analysed the data. KHC, RO'C, DRS, KR, MWD, MV, AH, BY, RS, MKCN and ACK interpreted the data. KHC, RO'C, LSH, RS, MKCN and ACK were responsible for drafting the article. All authors revised the manuscript and approved the final version.

\section{References}

1. Gordois A, Scuffham P, Shearer A (2003) The health care cost of diabetic peripheral neuropathy in the US. Diabetes Care 26:1790-1795
2. Tongers J, Roncalli J, Losordo D (2008) Therapeutic angiogenesis for critical limb ischemia: microvascular therapies coming of age. Circulation 118:9-16

3. Rajamani K, Colman P, Li L et al (2009) Effect of fenofibrate on amputation events in people with type 2 diabetes mellitus (FIELD study): a prespecified analysis of a randomised controlled trial. Lancet 373:1780-1788

4. Ohnaka K, Kono S (2010) Bilirubin, cardiovascular diseases and cancer: epidemiological perspectives. Expert Rev Endocrinol Metab 5:891-904

5. Breimer L, Mikhailidis D (2011) Is bilirubin a marker of vascular disease and/or cancer and is it a potential therapeutic target? Curr Pharm Des 17:3644-3655

6. Lin J, Vitek L, Schwertner H (2010) Serum bilirubin and genes controlling bilirubin concentrations as biomarkers for cardiovascular disease. Clin Chem 56:1535-1543

7. Stocker R, Perrella M (2006) Heme oxygenase-1: a novel drug target for atherosclerotic diseases? Circulation 114:2178-2189

8. Chan K, Ng M, Stocker R (2011) Haem oxygenase-1 and cardiovascular disease: mechanisms and therapeutic potential. Clin Sci (Lond) 120:493-504

9. Kawamura K, Ishikawa K, Wada Y et al (2005) Bilirubin from heme oxygenase-1 attenuates vascular endothelial activation and dysfunction. Arterioscler Thromb Vasc Biol 25:155-160

10. Stocker R, Yamamoto Y, McDonagh A, Glazer A, Ames B (1987) Bilirubin is an antioxidant of possible physiological importance. Science 235:1043-1046

11. Stocker R, Glazer A, Ames B (1987) Antioxidant activity of albumin-bound bilirubin. Proc Natl Acad Sci USA 84:5918-5922

12. Inoguchi $\mathrm{T}$, Sasaki S, Kobayashi K, Takayanagi R, Yamada $\mathrm{T}$ (2007) Relationship between Gilbert Syndrome and prevalence of vascular complications in patients with diabetes. JAMA 12:1398-1400

13. Tapan S, Dogru T, Tasci I, Ercin C, Ozgurtas T, Erbil M (2009) Soluble CD40 ligand and soluble P-selectin levels in Gilbert's syndrome: a link to protection against atherosclerosis? Clin Biochem 42:791-795

14. Vitek L, Jirsa M, Brodanova M et al (2002) Gilbert syndrome and ischemic heart disease: a protective effect of elevated bilirubin levels. Atherosclerosis 160:449-456

15. Abraham N, Kappas A (2008) Pharmacological and clinical aspects of heme oxygenase. Pharmacol Rev 60:79-127

16. Stocker R, Keaney JF (2004) Role of oxidative modifications in atherosclerosis. Physiol Rev 84:1381-1478

17. Breimer L, Spyropolous K, Winder A, Mikhailidis D, Hamilton G (1994) Is bilirubin protective against coronary artery disease? Clin Chem 40:1987-1988

18. Rantner B, Kollerits B, Anderwald-Stadler M et al (2008) Association between the UGT1A1 TA-repeat polymorphism and bilirubin concentration in patients with intermittent claudication: results from the CAVASIC study. Clin Chem 54:851-857

19. Perlstein T, Pande R, Beckman J, Creager M (2008) Serum total bilirubin level and prevalent lower-extremity peripheral arterial disease: National Health and Nutrition Examination Survey (NHANES) 1999 to 2004. Arterioscler Thromb Vasc Biol 28:166-172

20. Wang H, Han P, Zhang W et al (2012) Serum bilirubin level is negatively correlated with disease progression of peripheral arterial disease: an observational cohort study. Angiology 63:248-253

21. Johannesson A, Larsson G, Ramstrand N, Turkiewicz A, Wiréhn A, Atroshi I (2009) Incidence of lower-limb amputation in the diabetic and nondiabetic general population: a 10 -year populationbased cohort study of initial unilateral and contralateral amputations and reamputations. Diabetes Care 32:275-280

22. The FIELD Study Investigators (2005) Effects of long-term fenofibrate therapy on cardiovascular events in 9795 people with type 2 
diabetes mellitus (the FIELD study): randomised controlled trial. Lancet 366:1849-1861

23. Barrett-Connor E, Orchard T (1985) Diabetes and heart disease. In: National Diabetes Data Group. Diabetes in America: diabetes data compiled 1984. US Department of Health and Human Services, Washington, pp XVI, 1-41

24. Nakayama K (1995) Differences between enzymatic and diazo methods for measuring direct bilirubin. Eur J Clin Chem Clin Biochem 33:513-517

25. Selvin E, Erlinger T (2004) Prevalence of and risk factors for peripheral arterial disease in the United States: results from the National Health and Nutrition Examination Survey, 1999-2000. Circulation 110:738-743

26. Pasta D (2009) Learning when to be discrete: continuous vs. categorical predictors. SAS Glob Forum Pap 248:1-10

27. Djousse L, Levy D, Cupples L, Evans J, D’Agostino R, Ellison R (2001) Total serum bilirubin and risk of cardiovascular disease in the Framingham offspring study. Am J Cardiol 87:1196-1200

28. Ekblom K, Marklund S, Jansson J et al (2010) Plasma bilirubin and UGT1A $1 * 28$ are not protective factors against first-time myocardial infarction in a prospective, nested case-referent setting. Circ Cardiovasc Genet 3:340-347

29. Schwertner H, Jackson W, Tolan G (1994) Association of low serum concentration of bilirubin with increased risk of coronary artery disease. Clin Chem 40:18-23

30. Breimer L, Wannamethee G, Ebrahim S, Shaper A (1995) Serum bilirubin and risk of ischemic heart disease in middle-aged British men. Clin Chem 41:1504-1508

31. Hopkins P, Wu L, Hunt S, James B, Vincent M, Wiliams R (1996) Higher serum bilirubin is associated with decreased risk for early familial coronary artery disease. Arterioscler Thromb Vasc Biol $16: 250-255$

32. Madhavan M, Wattigney W, Srinivasan S, Berenson G (1997) Serum bilirubin distribution and its relation to cardiovascular risk in children and young adults. Atherosclerosis 131:107-113

33. Temme E, Zhang J, Schouten E, Kesteloot H (2001) Serum bilirubin and 10-year mortality risk in a Belgian population. Cancer Causes Control 12:887-894

34. Bosma P, van der Meer IM, Bakker C, Hofman A, PaulAbrahamse M, Witteman J (2003) UGT1A1*28 allele and coronary heart disease: the Rotterdam Study. Clin Chem 49:1180-1181

35. Novotny L, Vitek L (2003) Inverse relationship between serum bilirubin and atherosclerosis in men. A meta-analysis of published studies. Exp Biol Med 228:568-571

36. Lin J, O'Donnell C, Schwaiger J et al (2006) Association between the UGT1A $1 * 28$ allele, bilirubin levels, and coronary heart disease. Circulation 114:1476-1481

37. Troughton J, Woodside J, Young I et al (2007) Bilirubin and coronary heart disease risk in the Prospective Epidemiological
Study of Myocardial Infarction (PRIME). Eur J Cardiovasc Prev Rehabil 14:79-84

38. Chen Y, Chau L, Chen J, Lin S (2008) Serum bilirubin and ferritin levels link heme oxygenase-1 gene promoter polymorphism and susceptibility to coronary artery disease in diabetic patients. Diabetes Care 31:1615-1620

39. Kimm H, Yun J, Jo J, Jee S (2009) Low serum bilirubin level as an independent predictor of stroke incidence: a prospective study in Korean men and women. Stroke 40:3422-3427

40. Erdogan D, Gullu H, Yildirim E et al (2006) Low serum bilirubin levels are independently and inversely related to impaired flowmediated vasodilation and increased carotid intima-media thickness in both men and women. Atherosclerosis 184:431-437

41. Vítek L, Novotny L, Sperl M, Holaj R, Spacil J (2006) The inverse association of elevated serum bilirubin levels with subclinical carotid atherosclerosis. Cerebrovasc Dis 21:408-414

42. Maruhashi T, Soga J, Fujimura N et al (2012) Hyperbilirubinemia, augmentation of endothelial function, and decrease in oxidative stress in Gilbert syndrome. Circulation 126:598-603

43. Öllinger R, Bilban M, Erat A et al (2005) Bilirubin: a natural inhibitor of vascular smooth muscle cell proliferation. Circulation 112:1030-1039

44. Tenhunen R, Marver H, Schmid R (1968) The enzymatic conversion of heme to bilirubin by microsomal heme oxygenase. Proc Natl Acad Sci USA 61:748-755

45. Endler G, Exner M, Schillinger M et al (2004) A microsatellite polymorphism in the heme oxygenase-1 gene promoter is associated with increased bilirubin and HDL levels but not with coronary artery disease. Thromb Haemost 91:155-161

46. Lublinghoff N, Winkler K, Winkelmann B et al (2009) Genetic variants of the promoter of the heme oxygenase- 1 gene and their influence on cardiovascular disease (the Ludwigshafen Risk and Cardiovascular Health study). BMC Med Genet 10:36

47. Gajdos V, Petit F, Perret C et al (2006) Further evidence that the UGT1A1*28 allele is not associated with coronary heart disease: the ECTIM Study. Clin Chem 52:2313-2314

48. Oda E (2010) Bilirubin is negatively associated with A1C independently of fasting plasma glucose, age, obesity, inflammation, hemoglobin, and iron in apparently healthy Japanese men and women. Diabetes Care 22:e131

49. Ohnaka K, Kono S, Inoguchi T et al (2010) Inverse associations of serum bilirubin with high sensitivity C-reactive protein, glycated hemoglobin, and prevalence of type 2 diabetes in middle-aged and elderly Japanese men and women. Diabetes Res Clin Pract $88: 103-110$

50. Ames B, Cathcart R, Schwiers E, Hochstein P (1981) Uric acid provides an antioxidant defense in humans against oxidant- and radical-caused aging and cancer: a hypothesis. Proc Natl Acad Sci U S A 78:6858-6862 